\title{
INTOXICAÇÃO POR DISOFENOL EM CAPRINOS
}

\author{
POISONING BY DISOPHENOL IN GOATS
}

\section{Mauro Pereira Soares ${ }^{1}$ Fernando Sergio Castilhos Karam ${ }^{2}$ Gisele Braziliano de Andrade ${ }^{3}$}

\section{-RELATO DE CASO-}

\section{RESUMO}

Cinqüenta e seis cabras morreram em aproximadamente 44 horas após serem medicadas com disofenol. Dois animais foram necropsiados. Pontos de coloração marrom claro, com aproximadamente $2 \mathrm{~cm}$ de diâmetro foram observados na cápsula e superfície de corte do órgão. A superfície de corte dos rins apresentaram áreas claras intercaladas com áreas escuras. A bexiga urinária estava repleta com urina e sedimento. A cavidade abdominal, torácica e o saco pericárdico tinham acúmulo de fluído amarelado. Espuma foi encontrada na traquéia e pulmões. Metabolização da gordura coronariana e mesentérica também foi observada. As lesões histológicas caracterizaram-se por necrose centrolobular ou massiva do fígado; necrose do epitélio dos túbulos, com presença de cilindros e congestão nos rins; e edema do pulmão. Em quatro cabras, foi aplicado por via subcutânea disofenol a doses de 10, 10,5, 19,04 e 29,43mg por kg de peso vivo. $O$ caprino que recebeu $10,5 \mathrm{mg} / \mathrm{kg}$ foi mantido em uma sala à temperatura de aproximadamente $28^{\circ} \mathrm{C}$ durante 18 horas. O caprino que recebeu $29,43 \mathrm{mg} / \mathrm{kg}$ morreu. Os achados macroscópicos e histológicos foram similares aos observados na intoxicação espontânea.

Palavras-chave: disofenol, intoxicação, cabras.

\section{SUMMARY}

Fifty-six goats died approximately 44 hours after been medicated with disophenol. Two animals were necropsied. Brown spots of approximately $2 \mathrm{~cm}$ were observed in the capsule and cut surface of the liver. The cut surface of the kidneys presented clear areas mixed with dark areas. The urinary bladder was replete with urine and sediment. The abdominal, toracic and pericardic cavities had accumulation of yellow fluid. Edema was found in the trachea and lung. Metabolization of the coronary and mesenteric fat was also observed. Histologic lesions were characterized by centrilobular or diffuse necrosis of the liver; necrosis of the epitelial tubules, with presence of cilinders and congestion in the kidneys; and edema of the lung. Four goats were inoculated subcutaneously with 10, 10.5, 19.04 and 29.43mg per $\mathrm{kg}$ body weight of disophenol. The animal treated with $10.5 \mathrm{mg} / \mathrm{kg}$ was mantained during 18 hours at room with temperature of $28^{\circ} \mathrm{C}$. The goat that recieved $29.43 \mathrm{mg} / \mathrm{kg}$ died. The macroscopic and histologic findings were similar to those observed in the spontaneous case.

Key words: disophenol, poisoning, goat.

\section{INTRODUÇÃO}

Disofenol (2,6-diiodo-4-nitrofenol ou $\mathrm{C}_{6} \mathrm{H}_{3} \mathrm{I}_{2} \mathrm{NO}_{3}$ ) se apresenta na forma de finíssimos cristais de cor amarela, e caracteriza-se por ser pouco solúvel em água, mas fracamente solúvel em álcool. Apresenta toxicidade e estrutura química semelhante aos dinitrofenóis que são utilizados como herbicidas, sendo assim freqüentemente comparados (LIAO \& OEHME, 1980). É um produto à base de fenol, utilizado como anti-helmíntico por via subcutânea na dose de $10 \mathrm{mg} / \mathrm{kg}$. A margem de segurança é de três a quatro vezes a dose terapêutica (BOOTH \& McDONALD, 1992), porém intoxicações ocasionais podem ocorrer com doses menores (LIAO \& OEHME, 1980).

Após a aplicação, é rapidamente absorvido e parece acumular-se no plasma. Somente

\footnotetext{
${ }^{1}$ Médico Veterinário, Mestre, Laboratório Regional de Diagnóstico, Faculdade de Veterinária, Universidade Federal de Pelotas (UFPEL). Campus Universitário, CP 354, 96010-900 - Pelotas, RS. E-mail: mpsoares@ufpel. tche.br. Autor para correspondência.

${ }^{2}$ Médico Veterinário, aluno do Curso de Pós-graduação em Medicina Veterinária da Faculdade de Veterinaria da UFPEL.

${ }^{3}$ Médico Veterinário, Doutor, Professor Adjunto 1, Faculdade de Veterinária, UFPEL.
} 
uma pequena porção do produto é excretada na urina durante as primeiras 24 horas da administração. A dose terapêutica provoca aumentos nas freqüências cardíaca e respiratória e na temperatura corpórea, aparentemente causados pelo efeito da droga que atua como um desagregador da fosforilação oxidativa (BOOTH \& McDONALD, 1992). Para ser excretado, sofre biotransformação primária no fígado, por conjugação com glicuronídeos, através das oxidases de função mista, que têm a maior atividade enzimática nos hepatócitos das regiões centro-lobulares (CARLTON \& McGAVIN, 1998), e a primeira excreção ocorre, via renal (OSWEILER, 1998).

Animais intoxicados por fenóis apresentam, logo após a exposição ao produto, dor intensa, tremores musculares, incoordenação, hipertermia, sudorese, taquicardia, polipnéia, e rigor mortis precoce nos casos fatais (OSWEILER, 1998; LARINI, 1987; PENUMARTHY et al., 1975). Sinais adicionais como desidratação, acidose metabólica e anoxia são, também, observados (LEGENDRE, 1973; LIAO \& OEHME, 1980). A morte geralmente ocorre por insuficiência respiratória e cardíaca (LARINI, 1987). Em ruminantes, os metabólitos desses compostos acarretam meta-hemoglobinemia, hipoproteinemia e hemólise (RADOSTITS, 1994).

Os achados de necropsia incluem hemorragias no trato gastrointestinal e no timo, necrose hemorrágica centrolobular e congestão do fígado, ocasional congestão dos pulmões e rins, hiperemia do omento, mesentério e vesícula biliar e urina sanguinolenta (LIAO \& OEHME, 1980; PENUMARTHY et al., 1975).

Microscopicamente, ocorre necrose centrolobular multifocal aguda e necrose tubular renal. Nas intoxicações via oral, é possível observar necrose de coagulação nas mucosas (JONES \& HUNT, 1983; OSWEILER, 1998)

\section{RELATO DO CASO}

$\mathrm{Na}$ rotina do Laboratório Regional de Diagnóstico (LRD), da Faculdade de VeterináriaUFPel, foram recebidos dois caprinos que haviam sido medicados, via subcutânea, com uma solução de disofenol a 20\%, e vieram a óbito aproximadamente em 44 horas após a aplicação. No dia da aplicação do medicamento, segundo fontes da Estação Agroclimatológica da Embrapa CPACT de Pelotas/RS, os dados agroclimatológicos foram os seguintes: temperatura mínima $14,4^{\circ} \mathrm{C}$; temperatura máxima $26,8^{\circ} \mathrm{C}$; temperatura média diária $19,2^{\circ} \mathrm{C}$ e velocidade do vento a $2 \mathrm{~m}$ de altura $\left(\mathrm{m}^{\mathrm{s}-1}\right) \quad 0,8$. Segundo o proprietário, foi aplicado nos animais até
$20 \mathrm{~kg}, 1 \mathrm{ml}$ do produto e nos animais maiores 2 a $3 \mathrm{ml}$. Quatorze horas após a aplicação, já havia animais mortos. Os demais encontravam-se apáticos, não se alimentavam, alguns berravam continuamente, e os animais que se encontravam em pé, ao serem movimentados pareciam piorar e caíam em decúbito, não levantando mais e morrendo posteriormente. Após cerca de 44 horas, restaram apenas 4 animais de um rebanho de 60 caprinos. Destes, dois foram enviados para o LRD. Um deles, quase em coma, foi sacrificado (Caprino 1). O outro foi mantido em observação e apresentou decúbito esternal por longos períodos, evitando movimentarse o que fazia somente se fosse estimulado. Quando movimentado exibia sinais de dor com arqueamento do dorso. Apresentou ainda anorexia, depressão, dificuldade de micção e urina com abundante quantidade de sedimento. Em, aproximadamente, 108 horas após a medicação, este animal morreu (Caprino 2). Na necropsia, ambos os animais apresentaram o fígado com múltiplos focos de coloração marrom clara de, aproximadamente, $2 \mathrm{~cm}$ de diâmetro, tanto na superfície do órgão como no interior do parênquima. Os rins exibiam o parênquima com áreas claras intercaladas com áreas escuras. A bexiga apresentava-se com grande quantidade de urina e abundante sedimento. A cavidade abdominal, o saco pericárdico e a cavidade torácica possuíam acúmulo de líquido amarelado. No pulmão e traquéia, foi encontrado espuma. Havia mobilização da gordura coronariana e mesentérica. Os achados histopatológicos do fígado caracterizaram-se por necrose centrolobular à massiva dos hepatócitos. Os rins apresentaram necrose das células epiteliais dos túbulos, presença de cilindros hialinos na luz tubular e congestão. Nos pulmões, havia edema. No caprino 2, encontrou-se ainda, no fígado, fibrose periportal, proliferação de células dos ductos biliares e infiltrado de células inflamatórias mononucleares, e nos rins, áreas de calcificação. $\mathrm{Na}$ tentativa de reproduzir experimentalmente a intoxicação, utilizaram-se quatro caprinos com 2.100 ovos de parasitas gastrointestinais por grama de fezes e pesos de 32, 19, 21, e 26,5kg (respectivamente caprinos $\mathrm{n}^{\mathrm{o}} 3,4,5$ e 6 ). O produto usado foi retirado do mesmo frasco utilizado pelo proprietário. A dose terapêutica recomendada pelo fabricante é de $10 \mathrm{mg} / \mathrm{kg}$. No experimento, doses de $10,10,5,19,04$ e $29,43 \mathrm{mg} / \mathrm{kg}$ foram administradas via subcutânea respectivamente aos caprinos de $\mathrm{n}^{\mathrm{os}}$ $3,4,5$ e 6 . Todos os animais foram mantidos em temperatura ambiente, exceto o caprino 4 , que foi mantido à temperatura de aproximadamente $28^{\circ} \mathrm{C}$ durante 18 horas. Após 24 horas da aplicação, apenas o caprino 6 morreu. Os achados de necropsia 
e histopatológicos deste animal foram similares aos encontrados nos caprinos 1 e 2, porém, não havia fibrose hepática como a observada no caprino 2 .

\section{DISCUSSÃO}

Apesar do disofenol ser indicado como um produto com larga margem de segurança, 3 a 4 vezes superior à dose terapêutica (BOOTH \& McDONALD, 1992), algumas intoxicações podem ocorrer.

Com o objetivo de reproduzir experimentalmente os casos espontâneos, o caprino 6 recebeu a dosagem de $29,43 \mathrm{mg} / \mathrm{kg}$, (< que 3 vezes a dose terapêutica) e morreu 24 horas após. O caprino 5, que recebeu $19,04 \mathrm{mg} / \mathrm{kg}$, menos que o dobro da dose terapêutica, não morreu. Esses fatos sugerem que a dose tóxica do produto é menor que a margem de segurança indicada, e com isso, erros por super dosagem são facilmente alcançados por superestimação do peso dos animais.

As intoxicações por disofenol, geralmente, estão ligadas a fatores como elevação da temperatura ambiental e exercício físico, uma vez que os próprios compostos derivados do fenol incrementam o metabolismo energético celular e a exigência de oxigênio (OSWEILER, 1998; LEGENDRE, 1973). Com a dose terapêutica correta (10mg/kg), LEGENDRE (1973) provocou a intoxicação em um cão Irish Wolfhound que, após a medicação, foi exercitado durante 2 a 3 horas em jogos, num dia em que a temperatura ambiente ultrapassava os $32^{\circ}$ C. Após 5 horas, esse cão mostrou-se ofegante, com taquicardia e taquipnéia, mucosas extremamente hiperêmicas e temperatura retal de $41,4^{\circ}$ C. No presente experimento, a elevação da temperatura e a dosagem de $10,5 \mathrm{mg} / \mathrm{kg}$ (Caprino 4) não causaram a morte do animal. No caso de campo, o proprietário informou que, após a aplicação do produto, os animais foram colocados em ambiente fechado, para passarem a noite, e a temperatura ambiental, na ocasião, estava elevada. Segundo LARINI (1987), o aumento do metabolismo basal é proporcional à quantidade do produto no organismo, podendo atingir valores críticos quando a produção de calor exceder à dissipação fisiológica, provocando assim uma hipertermia letal. A exacerbação do metabolismo ocorre pelo desacoplamento da fosforilação oxidativa (OSWEILER, 1998) e explica a morte agônica dos animais relatada pelo proprietário, pois, se os animais eram movimentados, o quadro clínico parecia piorar e os que caíam, não levantavam mais e, posteriormente, morriam. A fosforilação oxidativa alterada leva à inibição do metabolismo energético, causando depleção dos níveis de ATP, acentuando a lesão química, ocasionando a morte celular (OSWEILER, 1998). Com a carência repentina de energia no organismo, há aumento da respiração para compensar a deficiência de ATP, causando acentuado consumo de oxigênio e diminuição das reservas de glicose.

Nos animais necropsiados, as alterações hepáticas encontradas são similares às observadas por OSWEILER (1998) e também coincidem com as observadas na histopatologia do caprino 1,2 e 6 . A fibrose hepática observada no caprino 2 é, provavelmente, um achado incidental. Já as alterações histológicas renais incluíram nefrose tubular e congestão, coincidindo com os achados de OSWEILER (1998) e PENUMARTHY $\boldsymbol{e t} \boldsymbol{a l}$. (1975). A presença de sedimentos na urina reflete a lesão renal, coincidindo com os achados dos autores acima citados e com LEGENDRE (1973).

No presente relato, o conjunto de dados epidemiológicos, os sinais clínicos, os achados macroscópicos e histopatológicos e a reprodução experimental permitiram realizar o diagnóstico da intoxicação.

\section{REFERÊNCIAS BIBLIOGRÁFICAS}

BOOTH, N.H., McDONALD, L.E. Farmacologia e terapêutica em veterinária. 6. ed. Rio de Janeiro : Guanabara Koogan, 1992. Cap.55: Drogas usadas contra nematódeos: p.743-744.

CARLTON, W.W., McGAVIN, M.D. Patologia veterinária especial de Thomson. Porto Alegre : Artes Médicas, 1998. $672 \mathrm{p}$.

JONES, T.C., HUNT, R.D. Veterinary pathology. 5 ed. Philadelphia : Lea \& Febiger, 1983. 1792p.

LARINI, L. Toxicologia. São Paulo : Manole, 1987. 315p.

LEGENDRE, A.M. Disophenol toxicosis in a dog. Journal of the American Veterinary Medical Association. v.163, n.2, p.149-150, 1973.

LIAO, J.T.F., OEHME, F.W. Literature Rewiews of phenolic compounds, III, disophenol. Veterinary and Human Toxicology. v.22, n.5, p. 315-317, 1980.

OSWEILER, G.D. Toxicologia veterinária. Porto Alegre : Artes Médicas, 1998. 526p.

PENUMARTHY, L., FREDERICH, W.O., MONTY, J.M. Investigations of therapeutic measures for disophenol toxicosis in dogs. American Journal of Veterinary Research. v.36, n.8, p.1259-1262, 1975.

RADOSTITS, M.O., BLOOD, D.C., GAY, C.C. Veterinary medicine. 8. ed. London: Baliére Tindal, 1994. p.1517.

Ciência Rural, v. 31, n. 1, 2001. 